\title{
PROJET DE CENTRE DE RECHERCHES \\ ASTROMETRIQUES ET GEODYNAMIQUES EN FRANCE
}

\author{
J. KOVALEVSKY \\ Observatoire de Meudon, France
}

\begin{abstract}
Résumé. Il y a de nombreux avantages de rassembler, en un même site, un grand choix d'instruments divers en astrométrie et géodynamique, en particulier pour étudier et comprendre les erreurs systématiques de diverses méthodes.

C'est une des raisons pour lesquelles un nouvel observatoire est en train d'être installé près de Grasse.

Le site choisi est le meilleur pour les observations aux astrolabes Danjon. Il entrera en fonction en 1974 et comportera deux astrolabes, un laser pour satellites, un télescope de Schmidt destiné aux observations astrométriques et, plus tard, un laser-lune et d'autres instruments.
\end{abstract}

\begin{abstract}
There are many advantages in having at the same site, a large array of various instruments for astrometry and geodynamics, in particular in order to provide a good insight into the systematic errors of various methods. This is one of the reasons why a new observational center is being equipped now near Grasse. The site chosen is the best found for Danjon astrolabes. It will start its operation in 1974 and will include two astrolabes, a satellite laser, a Doppler satellite receiving station, a Schmidt telescope fit for astrometric observations and, later, a lunar laser and other equipment.
\end{abstract}

Au cours de ce symposium, on aura discuté de l'apport considérable que l'on peut attendre, dans le domaine de l'astrométrie, des nouvelles méthodes d'observation. Qu'il s'agisse des instruments classiques comme l'instrument méridien ou l'astrolabe, rendus plus performants et automatisés ou qu'il s'agisse de techniques entièrement nouvelles comme le laser-lune, l'interférométrie à longue base ou des satellites spécialisés dans des mesures géodynamiques ou astrométriques, deux problèmes fondamentaux se posent:

(1) Il est nécessaire de prévoir une longue période de recouvrement des observations afin d'assurer la continuité des paramètres observés (mouvement du pôle ou rotation de la terre par exemple); les observations par les deux méthodes doivent être simultanées et être faites dans des conditions aussi voisines que possible, idéalement au même endroit.

(2) Les divers moyens d'observation sont évidemment grevés plus ou moins d'erreurs systématiques. Il est nécessaire d'analyser ces erreurs et, pour cela, comparer les résultats obtenus à partir de techniques différentes. Là encore, les comparaisons se feront dans les meilleures conditions si les instruments sont situés au même lieu.

C'est pourquoi, il apparait qu'il y a un gros avantage, de regrouper dans un observatoire unique l'ensemble des moyens modernes d'observation astrométrique, tout en les faisant voisiner avec des instruments classiques.

Sous l'impulsion d'un certain nombre d'astronomes français, notamment de $\mathbf{M}$. J. Delhaye, actuellement Directeur de l'Institut National d'Astronomie et de Géophysique (INAG), il a été décidé de consacrer une partie des moyens d'équipement vers une rénovation de l'astrométrie. Parallèlement, d'importants moyens spatiaux ont été mis, par le Centre National des Etudes Spatiales (CNES), à la disposition des 
astronomes et géodésiens français pour poursuivre des études de géodynamique spatiale, relatives surtout au potentiel terrestre, à la géodésie, aux divers mouvements de la terre et de sa croûte, et à la position de la lune.

Ces conditions favorables ont permis de tenter de créer un centre astronomique nouveau, essentiellement consacré à l'astronomie de position et à la dynamique du système terre-lune, en y rassemblant le plus grand nombre possible d'instruments nouveaux pouvant contribuer à ces domaines et en y assurant, par la venue d'équipes suffisamment nombreuses et expérimentées, l'exploitation de ces instruments, leur intercomparaison et l'analyse des résultats d'observations.

C'est en 1965 qu'a commencé la recherche d'un site pour le nouvel observatoire. Il s'agissait alors, dans l'esprit des promoteurs, de trouver un endroit pour placer les instruments d'astronomie de position qui étaient tous situés dans des observatoires englobés dans d'importantes agglomérations urbaines (Paris, Bordeaux, Besançon, etc....) et de réaliser en quelque sorte, une opération analogue à celle qui avait été faite pour les instruments d'astrophysique à Saint Michel et au Pic-du-Midi. Il était en effet vite apparu que les qualités du ciel nécessaires pour l'astrophysique (clarté, scintillation faible, faible quantité de vapeur d'eau) étaient très différentes de celles que l'on recherche en astronomie de position (stabilité des images avec des périodes de l'ordre de quelques secondes, pas de réfraction anormale).

La qualité astronomique du ciel avait été testée par des séries d'observation à l'astrolabe. Huit sites ont ainsi été étudiés et comparés par F. Laclare qui a établi un 'rendement' de chaque station proportionnel à la fois à l'erreur probable des observations et au nombre de nuits. Un site s'est avéré être meilleur que les autres. Situé au nord de Grasse, à $50 \mathrm{~km}$ de Nice, à une altitude moyenne de 1300 mètres, le plateau de Calern a ainsi été choisi, en 1970, comme site du futur Centre d'Etudes et de Recherches Géodynamiques et Astronomiques (CERGA) (figure 1).

Dès la fin de 1970, une petite station est installée au Calern sous la responsabilité de F. Laclare et des observations régulières d'astrolabe Danjon y sont effectuées. La station horaire est équipée d'une horloge à césium et une station de réception Doppler pour satellites vient d'y être installée. Enfin dans une grotte voisine, deux pendules horizontaux du type Melchior, prétés par l'Observatoire d'Uccle ont été placés pour des mesures de marées terrestres tandis qu'une campagne de mesures par gravimètre a été effectuée en début d'année.

La qualité du site a été confirmée. Près de 230 nuits par an sont au moins partiellement utilisables pour l'observation sur des périodes continues d'au moins deux heures. L'erreur quadratique moyenne des observations à l'astrolabe est 1,2 fois plus faible qu'à Paris, avec les mêmes observateurs (figure 2).

C'est en été 1974, que le CERGA sera effectivement créé et qu'une trentaine de personnes venant surtout de Paris, viendront en compléter le personnel. Et, en 1974 et 1975 , une première tranche d'équipement instrumental sera mise en place. Les instruments suivants y seront alors installés.

$1^{\circ}$ Un second astrolabe impersonnel Danjon venant de l'observatoire de Paris(?).

$2^{\circ}$ Le prototype d'un astrolabe photo-électrique avec deux équerres optiques 


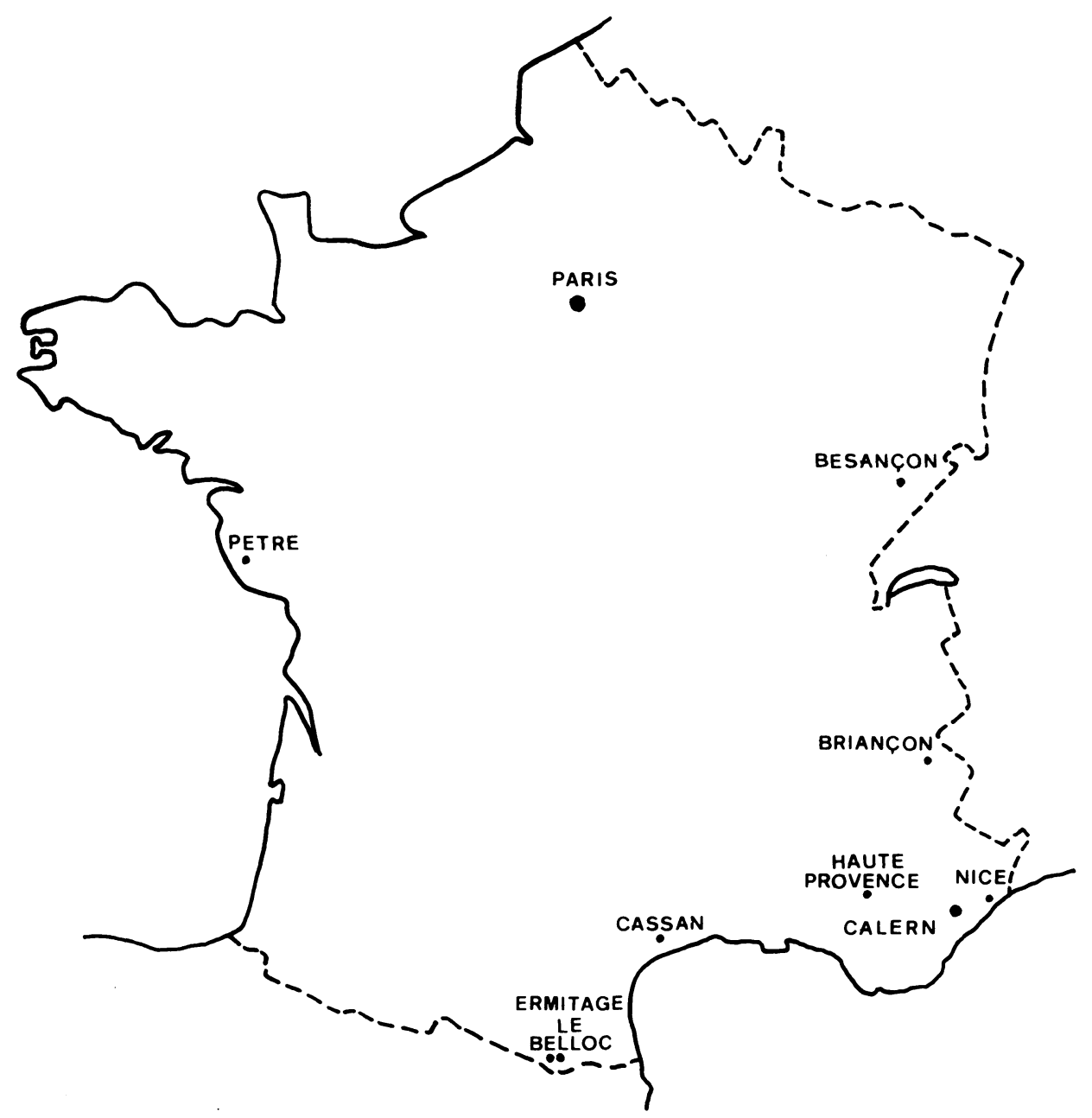

Fig. 1. Sites étudiés pour le choix d'un observatoire astronomique en France.

d'angles différents permettant des déterminations absolues de déclinaisons et atteignant la magnitude 7,5.

$3^{\circ}$ Un laser pour satellites artificiels, conçu pour observer de nuit en poursuite par visée optique ou de jour et de nuit par poursuite automatique, avec une précision de l'ordre de 20 à 30 centimètres sur les mesures de distance.

$4^{\circ}$ Un téléscope de $S c h m i d t$ avec un miroir de $150 \mathrm{~cm}$ et avec une lame de $90 \mathrm{~cm}$. L'optique a été étudiée de telle façon que le champ soit suffisamment proche d'un plan pour que l'on puisse prendre des clichés de $6^{\circ} \times 6^{\circ}$ sur des plaques de verre montées sur un chassis spécial. L'ouverture focale résultante étant de $F / 3,5$, on espère avoir une précision meilleure que 0,1 sur la position des étoiles. Ce télescope servira avant tout pour établir un catalogue de position des étoiles, comme première partie 
d'une nouvelle 'carte de ciel'. Il sera aussi utilisé pour l'observation de certains astres faibles du système solaire (satellites), mais aura plus tard, de plus en plus une vocation astrophysique. Une machine à mesurer automatique est actuellement étudiée pour réduire les clichés de ce téléscope.

$5^{\circ}$ Un laser-lune dont la précision de tir devrait être initialement de un mètre. Le laser et la partie électronique seront celles qui sont actuellement en place au Pic-duMidi, mais l'optique, étudiée à l'INAG sera nouvelle. Il s'agira d'un télescope d'environ $150 \mathrm{~cm}$ d'ouverture. Cet instrument travaillera de façon continue de jour comme de nuit chaque fois que cela sera possible.

(6) Un ensemble de gravimètre et de pendules horizontaux pour suivre les mouvements du sol de l'observatoire.

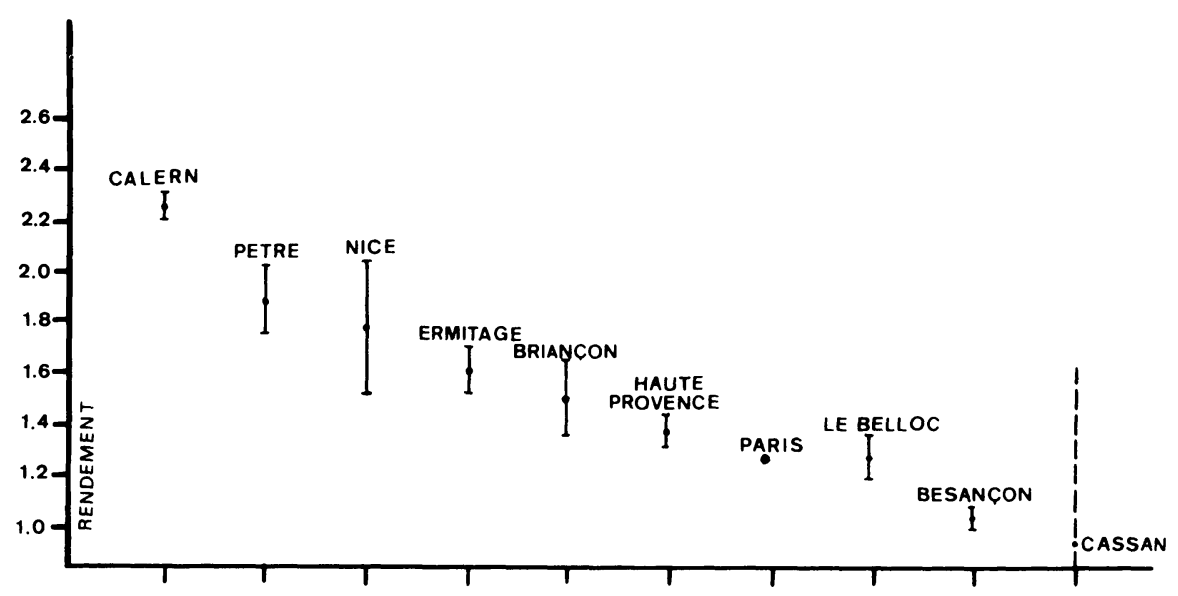

Fig. 2. Résultats de la prospection astronomique en France. Le rendement d'une station dépend de la qualité des images et du nombre de nuits d'observation par an.

(7) Un terminal relié à un grand calculateur Control Data/7700 du CNES à Toulouse et permettant donc de faire toutes les réductions aussi bien que des travaux théoriques.

Enfin, la proximité de l'observatoire de Nice $(50 \mathrm{~km})$ où se trouvent deux lunettes, permettra au personnel du CERGA de poursuivre des études des étoiles doubles et de sélénodésie déjà entreprises. Il est à noter, réciproquement, que les astronomes non résidents, qu'ils soient français ou étrangers, seront les bienvenus pour venir au CERGA faire des recherches dans les domaines des compétences scientifiques de ce centre.

Les projets pour un avenir plus lointain ne manquent pàs, mais aucune décision n'a encore été prise à leur sujet. L'effort sera poursuivi pour y mettre des instruments modernes, aussi automatisés que possible, susceptibles de compléter un ensemble destiné à améliorer par tous les moyens possibles la connaissance de la position et des mouvements des astres et contribuer à l'établissement d'un système de référence aussi précis que possible pour toutes les études de cinématique stellaire et de la dy- 
namique du globe terrestre, fondamentale dans la compréhension de sa structure interne.

Mais pour beaucoup des problèmes auquels nous voulons nous attaquer, notamment dans les domaines de la dynamique de la terre et de la lune et des systèmes de référence, une station aussi complètement équipée soit-elle ne suffit pas. Il serait souhaitable que plusieurs observatoires du type du CERGA soient installés dans le monde, bien distribués géographiquement, qui constitueraient une espèce de base fondamentale servant de référence terrestre aux mesures astrométriques. Les résultats obtenus par diverses techniques seront encore plus facilement comparables et la détection des erreurs systématiques sera encore plus sûre. Pour ne donner qu'un exemple, les différences systématiques constatées entre le mouvement du pôle déduit des observations des satellites TRANSIT peuvent avoir des origines très diverses, l'une étant le fait qu'ils s'appuient sur des observatoires presque tous différents. Une étude de cet effet possible n'est guère envisageable que si plusieurs stations sont simultanément équipées de récepteurs Doppler et d'astrolabes. C'est en ce sens que nous pensons que sera atteinte la plus grande efficacité astronomique des équipements coûteux qui sont mis en place un peu partout. L'expérience que ne manquera pas d'acquérir le CERGA sera, nous le souhaitons, réussie et pourra servir d'exemple dans d'autres régions du monde.

\section{DISCUSSION}

Luck: The Division of National Mapping, Australia is installing a Lunar Laser Ranger in the Australian Capital Territory, at an altitude of $1300 \mathrm{~m}$, within $1 \mathrm{~km}$ of an ongoing NASA satellite tracking station and within $40 \mathrm{~km}$ of the Mount Stromlo PZT, thus satisfying requirements of comparing classical and new techniques - in the southern hemisphere. A laser satellite tracking system might be added in the foreseeable future.

The lunar laser ranger is the 60 -in. AFCRL system with CerVit mirror formerly at Mount Lemmon, on indefinite loan from NASA through SAO.

Kovalevsky: I am very happy to see that such a center will exist in Australia and hope that we shall have some co-operative programmes.

Klock: What is your timetable for placing your proposed astrometric center into operation, and will you move the Bordeaux Transit Circle to the new site or construct a new instrument?

Kovalevsky: Most of the instrumentation quoted will be on the site in 1974 or 1975.

The project of a new Transit instrument for CERGA is not yet approved. The idea is to build a new instrument using Requième's techniques tested on the Bordeaux instrument.

Fracastoro: A new 42 in. astrometric telescope has been installed at the Observatory of Turin. 